\title{
GESTÃO DE TESES E DISSERTAÇÕES ELETRÔNICAS NO BRASIL: SOBRE BIBLIOTECAS DIGITAIS DE TESES E DISSERTAÇÕES E REPOSITÓRIOS INSTITUCIONAIS
}

\author{
GESTIÓN DE TESIS Y DISERTACIONES \\ ELECTRÓNICAS EN BRASIL: ACERCA DE \\ BIBLIOTECAS DIGITALES DE TESIS Y \\ DISERTACIONES Y REPOSITORIOS \\ INSTITUCIONALES
}

\begin{abstract}
Fernando César Lima Leite - fernandodfc@gmail.com Doutor em Ciência da Informação pela Universidade de Brasília (UnB). Professor da Faculdade de Ciência da Informação da UnB.

Tainá Batista de Assis - tbatista.assis@gmail.com Mestre em Ciência da Informação pela Universidade de Brasília (UnB). Coordenadora de Atendimento à Comunidade do Instituto Brasileiro de Informação em Ciência e Tecnologia (IBICT).
\end{abstract}

Bianca Amaro de Melo - bianca@ibict.br Doutora em Lingüística Aplicada pela Universidade Pompeu Fabra (Espanha). Coordenadora da Biblioteca Digital Brasileira de Teses e Dissertações (BDTD).

\section{RESUMO}

Introdução: Apresenta análise sobre a gestão das teses e dissertações eletrônicas no contexto brasileiro. Os dilemas da coexistência de bibliotecas digitais de teses e dissertações e repositórios institucionais e suas implicações são discutidos e possíveis caminhos a serem considerados por atores responsáveis por sua gestão em nível institucional e nacional são propostos.

Objetivo: O objetivo do artigo é apresentar e analisar implicações decorrentes da existência concomitante das bibliotecas digitais de teses e dissertações e RI como sistemas de gestão de teses e dissertações e, além disso, propor e discutir alternativas de solução aos problemas enfrentados por atores responsáveis por sua gestão em nível institucional e nacional.

Metodologia: Dados sobre o estado dos sistemas de gestão de teses e dissertações foram obtidos junto IBICT. A análise permitiu agrupar as 
instituições brasileiras em três grandes categorias que representam a situação atual da gestão de teses e dissertações no Brasil.

Resultados: Obtiveram-se as configurações de três grandes cenários e suas implicações institucionais e nacional para a gestão de teses e dissertações. Dessas configurações foram elencadas alternativas de soluções para os problemas identificados.

Conclusões: Entre as conclusões, destacam-se: a continuidade da Biblioteca Digital Brasileira de Teses e Dissertações do IBICT (BDTD) não implica, necessariamente, na continuidade das bibliotecas digitais de teses e dissertações. O investimento nos repositórios institucionais não significa o fim da BDTD; parte das universidades e instituições de pesquisa brasileiras não gozam de condições que as permitam manter efetivamente os dois sistemas.

Palavras-chave: Teses e dissertações. Teses e dissertações eletrônicas. BDTD. Bibliotecas digitais de teses e dissertações. Repositórios institucionais. RI. Acesso aberto.

\section{INTRODUÇÃO}

É possível considerar que o acesso aberto à informação científica no Brasil teve sua gênese marcada pela iniciativa SciELO em 1997, que mais tarde viria a incorporar - em certa medida - os pressupostos do acesso aberto, mas, sobretudo, na construção da Biblioteca Digital Brasileira de Teses e Dissertações (BDTD), em 2002.

Muito embora tais iniciativas tenham se desenvolvido sob as condições tecnológicas e políticas vigentes à época, é possível, hoje, compreender que ambas representam as duas principais estratégias de viabilização do movimento: a publicação de periódicos científicos de acesso aberto (via dourada) e a segunda o arquivamento da produção científica em repositórios digitais (via verde), respectivamente. Desde então, tanto a primeira quanto a segunda estratégia se desenvolveram substancialmente no país e imprimem especificidades sistêmicas e operacionais do movimento de acesso aberto no Brasil. Uma dessas peculiaridades diz respeito à propagação de repositórios institucionais de acesso aberto à informação científica $(\mathrm{RI})$ para gerenciar a produção científica em sua totalidade, incluindo teses e dissertações, em 
coexistência com as inúmeras bibliotecas digitais de teses e dissertações (BDT\&D), que fazem parte da BDTD.

Em princípio, tal situação não significaria problema algum se não fosse o fato de que teses e dissertações, por muitas razões, constituírem um dos primeiros e principais tipos de conteúdos dos RI. Se por um lado a existência concomitante desses dois tipos de sistemas de informação de acesso aberto reforça a gestão e comunicação da produção científica nacional, por outro apresenta desafios relacionados com a gestão da informação científica tanto para instituições que os mantém quanto para a formulação de políticas e constituição de redes em nível nacional.

Este artigo tem como objetivo apresentar e analisar implicações decorrentes da existência concomitante das BDT\&D e RI como sistemas de gestão de teses e dissertações e, além disso, propor e discutir alternativas de solução aos problemas enfrentados por atores responsáveis por sua gestão em nível institucional e nacional.

\section{FUNDAMENTAÇÃO TEÓRICA}

Do ponto de vista teórico, teses e dissertações são consideradas, ainda, literatura cinzenta. Em razão disso, é importante resgatar alguns conceitos que serão utilizados para sustentar reflexões e implicações sobre a gestão de teses e dissertações no Brasil.

A literatura cinzenta é caracterizada como sendo aquela literatura de difícil acesso, restrita a um pequeno grupo dentro de uma comunidade, não comercial e com pouco volume de reprodução. Ou seja, é o tipo de literatura cuja disseminação e acesso são limitados. Segundo Almeida (2000), trata-se documentos não convencionais, semipublicados, invisíveis e caracterizados por ter circulação restrita, assim como acesso e disponibilidade limitados. Segundo a autora, esse tipo de literatura possui algumas peculiaridades, tais como: 
- normas variáveis de produção e edição;

- controle bibliográfico ineficaz, uma vez que não se submete às agências de controle bibliográfico internacionais;

- dificuldade de uso, uma vez muitos desses documentos encontram-se em suportes como microfilmes e microfichas;

- concisão, uma vez que tem caráter menos detalhado que a literatura convencional, visto que, em princípio, não se tem a intenção de publicar;

- produção institucional, uma vez que, em geral, é de responsabilidade de organizações governamentais, universidades, centros de pesquisa.

Di Cesare e Sala (1995) elaboraram classificação da literatura cinzenta tendo por base o seu contraste com literatura convencional, também conhecida como branca. Ao adotarem gradações de cinza, as autoras consideraram que, quando mais claro o tom de cinza mais próxima da literatura convencional (branca) estaria a literatura cinzenta. Esta classificação foi representada por uma pirâmide (Figura 1):

Figura 1- Classificação da literatura cinzenta.

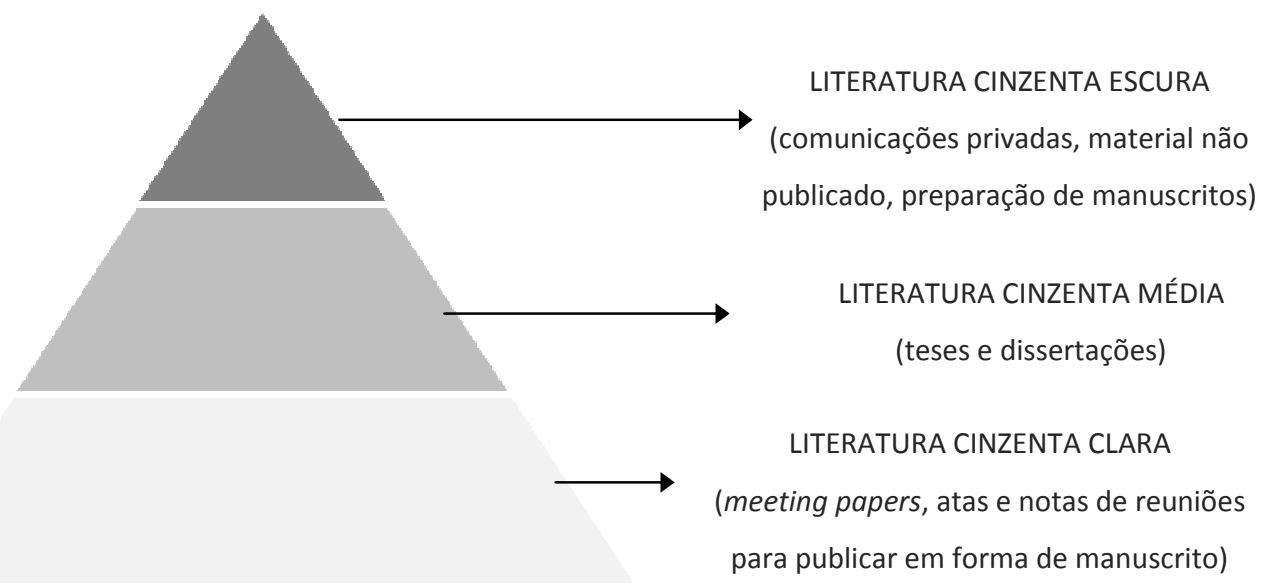

Fonte: Di Cesare e Sala (1995).

No entanto, o contexto de produção, distribuição e uso da literatura cinzenta sofreu profundas transformações na medida em que avanços tecnológicos foram adotados. Em razão dessas transformações, aspectos que definiam razões pelas quais 
determinados documentos consistem ou não em literatura cinzenta, como é o caso da dificuldade de acesso, circulação restrita e controle bibliográfico ineficaz, tornaram-se frágeis, especialmente a partir da década de 1990. Essa situação é claramente percebida no caso das teses e dissertações, classificadas por Di Cesare e Sala (1995) como literatura cinzenta média.

As teses e dissertações sofreram significativa modificação em sua função como canal de comunicação científica. Ou seja, na medida em que a tecnologia passou a ser utilizada na produção e, especialmente, na disseminação e promoção de acesso a resultados de pesquisa relatados em teses e dissertações, elas tornaram-se canais de comunicação que veiculam as descobertas, antes mesmo da literatura científica formalmente publicada. Isso foi possível graças ao surgimento de iniciativas de sistemas de informação digitais na internet com a finalidade de obter, organizar, armazenar, preservar, tornar recuperável e, sobretudo, disseminar de teses e dissertações em todo o mundo.

Normalmente tais sistemas dizem respeito às Bibliotecas Digitais de Teses e Dissertações (BDT\&D), que se dedicam exclusivamente à gestão e ampla disseminação de teses e dissertações de uma ou de um grupo de instituições, e Rl, que se dedicam à gestão e ampla disseminação da totalidade da produção científica de uma universidade ou instituto de pesquisa, incluindo as teses e dissertações. Em decorrência do surgimento do BDT\&D e Rl, diferentemente do que se considerava anteriormente, teses e dissertações estão associadas a processos que permitem:

- eficácia de seu registro, contribuindo significativamente para o controle bibliográfico em ambiente digital;

- facilidade de uso, uma vez que a disponibilidade e acessibilidade ao texto seu completo é promovida amplamente por sistemas de informação em ambiente digital e online;

- inserção do ciclo da comunicação científica. 
As teses e dissertações constituem o conjunto da produção científica de uma instituição, especialmente das universidades, onde está concentrada a quase totalidade dos programas de pós-graduação. Mais do que requisitos para a conclusão de cursos de mestrado e/ou doutorado e mais do que relatórios de pesquisa, em razão do desenvolvimento tecnológico dos sistemas de informação que as gerenciam, teses e dissertações passaram a trazer consigo relevante potencial de comunicação primária de resultados de investigação. Nesse sentido, tanto a pesquisa quanto o desenvolvimento de sistemas de informação de acesso aberto para a gestão de teses e dissertações, deve levar em conta os seguintes pressupostos:

- a informação científica a ser gerenciada por RI está contida principalmente em artigos de periódicos avaliados por pares, livros, capítulos de livros, anais de conferências e também teses e dissertações;

- dentre os tipos de documentos de interesse dos Rl, teses e dissertações constituem as unidades de comunicação científica mais transversais e de igual importância em todas as áreas do conhecimento (padrão em todas as áreas);

- por serem produzidas nos programas de pós-graduação (mestrado e doutorado) no âmbito de universidades e instituições de pesquisa, teses e dissertações prescindem da figura do editor para estarem acessíveis, ou seja, os direitos patrimoniais pertencem exclusivamente ao autor e são frequentemente cedidos, de maneira não exclusiva, às instituições onde foram produzidas (fácil gestão de direitos autorais);

- teses e dissertações há muito representam a parcela da produção científica institucional gerenciada pelas bibliotecas de pesquisa e universitárias, anteriormente em ambiente impresso e agora em ambiente digital.

\section{O ESTADO DA GESTÃO DE TESES E DISSERTAÇÕES NO BRASIL}

Teses e dissertações constituem os primeiros documentos a serem depositados em RI e são o carro-chefe de seu povoamento. Van 
Der Graaf e Van Eijndhoven (2008) apontaram que mais de $80 \%$ dos RI da União Européia contém teses e dissertações. No Directory of Open Access Repositories (OpenDOAR), é possível constatar que são o tipo de conteúdo mais frequente em repositórios digitais, ficando atrás somente dos artigos de periódicos, conforme ilustrado na Figura 2. Este fato é especialmente verdade no Brasil graças à BDTD, da qual 97 instituições, entre universidades e institutos de pesquisa, fazem parte atualmente ${ }^{1}$, contribuindo com mais de 260.078 teses e dissertações.

Figura 2 - Tipos de conteúdos em repositórios digitais

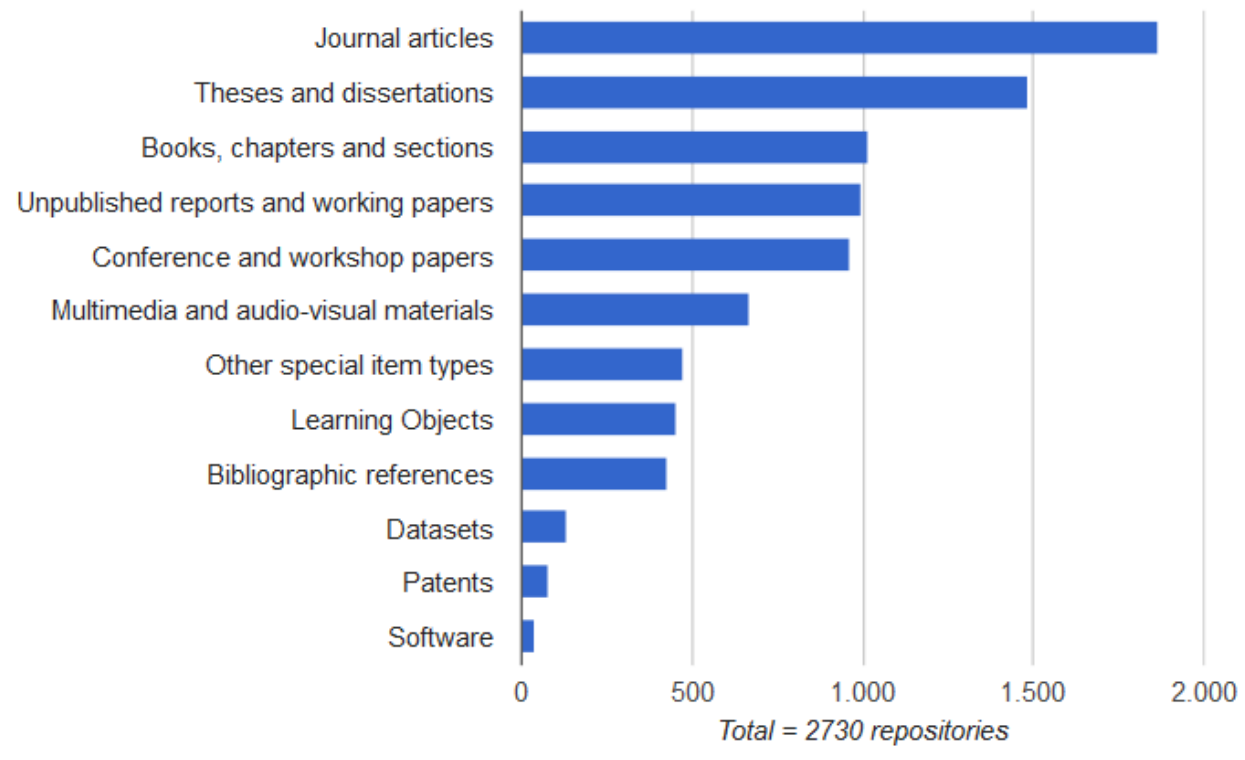

Fonte: OpenDOAR (2015).

A contribuição brasileira para 0 acervo mundial de teses e dissertações disponíveis e acessíveis em ambiente digital é significativa. O Diretório Open Access Theses and Dissertations - OATD (http://oatd.org/oatd/) aponta para um número de aproximadamente 2.422.068 teses e dissertações, provenientes de cerca de 1.000 instituições de todo o mundo ${ }^{2}$. O Brasil é responsável pela segunda

\footnotetext{
${ }^{1}$ Dado informado na página da BDTD.

2 Dados informados pelo OATD em março de 2015.
} 
maior coleção de teses e dissertações indexadas pelo OATD, alcançando a marca de 250.043 documentos $^{3}$, ficando atrás apenas dos Estados Unidos, de onde podem ser recuperadas 686.172 teses e dissertações. Essa significativa contribuição brasileira é decorrente dos esforços da BDTD, que resulta de esforços de inúmeras instituições brasileiras sob a coordenação do Instituto Brasileiro de Informação em Ciência e Tecnologia (IBICT).

De acordo com registros IBICT, o Brasil conta hoje com cerca de $40 \mathrm{RI}$, que são coletados pelo sistema OASISBR (http://oasisbr.ibict.br/), e 97 bibliotecas digitais de teses e dissertações, que fazem parte da Biblioteca Digital Brasileira de Teses e Dissertações. Ou seja, 137 instituições brasileiras possuem sistemas de informação de acesso aberto para gerenciar a produção científica nacional, sejam eles exclusivos para teses e dissertações ou que contemplam a produção científica como um todo. uma rápida análise do contexto desses sistemas utilizados para a gestão de teses e dissertações no brasil permitiu desenhar o seguinte cenário:

- 66 instituições possuem apenas BDT\&D;

- das 40 instituições que possuem ri, 29 também possuem biblioteca digital de teses e dissertações;

- 34 instituições possuem apenas RI. Desse conjunto de instituições, 29 possuem coleções de teses e dissertações em seus ri (5 não lidam com teses e dissertações);

- entre as 137 instituições, 9 delas possuem BDT\&D e RI ao mesmo tempo;

Da leitura desse cenário é possível afirmar que as instituições brasileiras podem ser agrupadas em um dos três grandes categorias

${ }^{3}$ A quantidade de T\&D brasileiras registradas pelo OATD é menor que quantidade real, visto que atualmente muitas instituições possuem RI que contemplam esse tipo de documento mas que não fazem parte da BDTD (sistema que fornece metadados das T\&D para o OATD). 
que representam a situação atual da gestão de teses e dissertações no Brasil. São eles:

1.Instituições que possuem RI e BDT\&D ao mesmo tempo e que participam da BDTD por meio destas últimas. Estas instituições podem ainda ser divididas entre três subgrupos:

- aquelas que alimentam os dois sistemas com teses $e$ dissertações (RI e BDT\&D);

- aquelas que alimentam somente as BDT\&D;

- aquelas que deixaram de alimentar as BDT\&D e alimentam apenas o RI.

2. Instituições que possuem apenas $\mathrm{RI}$ e gerenciam suas TESES $\mathrm{E}$ DISSERTAÇÕES por intermédio destes. Estas podem ser divididas em dois subgrupos:

- aquelas que participam da BDTD;

- aquelas que não participam da BDTD.

3. Instituições que possuem apenas BDT\&D e não gerenciam outros tipos de produção científica.

\subsection{Implicações das três situações da gestão de teses e dissertações}

Instituições presentes no Grupo 1

As instituições presentes no Grupo 1 têm enfrentado problemas decorrentes da coexistência dos RI e de suas bibliotecas digitais de teses e dissertações, dependendo ainda do subgrupo do qual fazem parte.

O primeiro problema a ser destacado diz respeito à duplicação de esforços para o depósito de teses e dissertações nos dois sistemas, visto que, aparentemente, parece não haver nenhuma instituição que tenha desenvolvido uma interface única de depósito para os dois sistemas. A alimentação duplicada não faz sentido algum se considerado o fato de que as finalidades dos RI e das BDT\&D são absolutamente convergentes. Outras dificuldades associadas são a dedicação de uma quantidade maior de pessoas, a manutenção institucional de dois sistemas, a manutenção do TEDE (sistema de teses 
e dissertações) pelo IBICT e prejuízo aos usuários que dispõem de duas interfaces para as mesmas atividades.

As instituições que tentam evitar parte dos problemas mencionados terminam por enfrentar outros. No segundo problema estão aquelas instituições que decidiram por não duplicar esforços e resolveram não alimentar seus $\mathrm{RI}$ com teses e dissertações e continuam alimentando normalmente suas BDT\&D. Se por um lado esta decisão pode evitar retrabalho, por outro a instituição promove a segmentação de sua produção científica. De um lado, no RI, estão artigos de periódicos, trabalhos publicados em anais de conferências, capítulos de livros, livros, etc. Do outro lado as teses e dissertações. Com isso, o RI deixa de reunir a totalidade da produção científica, restando ainda problemas de manutenção de dois sistemas e a penalização do usuário.

O terceiro problema que merece destaque é enfrentado por aquelas instituições que, apesar de possuírem os dois sistemas, para evitar os dissabores mencionados, decidiram alimentar exclusivamente o RI. Talvez a consequência desta decisão seja a mais preocupante: a não participação efetiva na BDTD. Com raras exceções no país, instituições que assim procedem passam a não ter, a partir de um determinado momento, os metadados de suas novas teses e dissertações coletadas pela BDTD e, consequentemente, não estão sendo coletadas pelo OATD e nem pela NDLTD.

\section{Instituições presentes no Grupo 2}

Há aquelas instituições que gerenciam suas teses e dissertações por meio dos próprios RI, o que é natural, pois estes conteúdos constituem a produção científica institucional. As instituições desse grupo encontram-se nessa situação por duas razões. A primeira é por nunca terem gerenciado suas coleções de teses e dissertações anteriormente, e o RI surgiu também para suprir esta necessidade (porém, não fazem parte da BDTD). A segunda é por nunca terem 
desenvolvido facilidade tecnológica para exposição de metadados de teses e dissertações a partir do próprio RI de modo que a BDTD ainda sim pudesse realizar a coleta. São poucos os casos de Rl cujas coleções de teses e dissertações são coletadas pela BDTD.

\section{Instituições presentes no Grupo 3}

São as instituições que, estrategicamente, requerem maior atenção dos esforços nacionais em favor do acesso aberto empreendidos, principalmente, pelo IBICT. Encontram-se nessa condição 57 instituições que possuem suas BDT\&D mas que, até o momento, segundo informações do próprio IBICT, não atentaram para a necessidade de gerenciar a produção científica em sua totalidade por meio de RI. A experiência prévia com as BDT\&D representa passos já percorridos na construção de RI, uma vez que muitos aspectos gerenciais e tecnológicos são bastante similares no desenvolvimento de ambos os sistemas.

\section{POSSÍVEIS CAMINHOS E CONSIDERAÇÕES FINAIS}

Tendo em vista que tanto os $\mathrm{Rl}$ quanto as BDT\&D constituem elementos de um sistema de gestão e comunicação da informação científica que não estão restritos às instituições ou países, a compreensão de seus problemas e as respostas a eles deve ocorrer sob uma perspectiva sistêmica. Por esta razão, nenhum esforço para tal deve considerar como parâmetro único as condições locais, sobretudo, aquelas relacionadas com as particularidades referentes às infraestruturas tecnológicas, organizacionais e mesmo políticas das instituições.

É importante frisar também que as instituições não são as únicas responsáveis pelos problemas mencionados ao longo deste artigo, nem tão pouco por suas soluções, até por estas dependerem, em todas as 
suas dimensões, de um norteamento sistêmico para que as tornem factíveis. Tanto a BDTD quanto a proliferação de RI no Brasil tem por trás esforços e articulação do IBICT, que é responsável nacionalmente pelas políticas de informação científica e tecnológica.

Diante das inúmeras facetas dos muitos problemas, qual é a condição que mais acomoda as diferentes situações? A resposta a esta pergunta não pode deixar de considerar que:

- RI nasceram para gerenciar e tornar acessível a totalidade da produção científica institucional e são uma realidade mundial;

- A BDTD surgiu em consonância com os avanços mundiais em relação às teses e dissertações eletrônicas, e, no Brasil, seu advento ocorre antes de qualquer iniciativa de Rl;

- Maior parte das universidades e instituições de pesquisa brasileiras não gozam de condições que as permitam manter efetivamente os dois sistemas;

- A quantidade de RI no Brasil tende a crescer cada vez mais;

- A continuidade da BDTD não implica, necessariamente, na continuidade das BDT\&D. O investimento nos RI não significa na não existência da BDTD;

- RI podem, com facilidade, assumir as funções das BDT\&D, enquanto estas, na maior parte dos casos, estão tecnologicamente impossibilitas de se transformarem em RI.

Nenhum dos problemas destacados deve ser resolvido única e exclusivamente a partir da perspectiva tecnológica. Embora as respostas passem necessariamente por esta dimensão, qualquer intervenção sistêmica deve ser precedida de análises de viabilidade organizacional, tecnológica e política nas instituições.

Partindo de todas as considerações feitas anteriormente, e tendo por base experiência de pesquisa e de desenvolvimento sobre acesso aberto e RI, sugere-se como ponto de partida para discussão a seguinte condição: o investimento nos $R I$ como os ambientes responsáveis pelo gerenciamento da totalidade da produção científica das universidades e instituições de pesquisa brasileiras e o fortalecimento da BDTD como agregador de teses e dissertações depositadas nos repositórios (seja 
como interface independente seja como conjunto de dados dentro do OASISbr).

Tal condição requer:

- A incorporação das funções desempenhadas pelas BDT\&D nos RI. Em termos tecnológicos significa a absorção das funções do TEDE (ou outro sistema adotado) pelo software utilizado no RI;

- Desenvolvimento de metodologia padrão de migração de dados das BDT\&D para os RI, incluindo análises de padrões de metadados e suporte;

- Adaptação/configuração, ou procedimento similar, para que os $\mathrm{RI}$ (que maior parte utiliza o DSpace) sejam capazes de expor adequadamente/separadamente metadados de teses $e$ dissertações;

- Adaptação/configuração, ou procedimento similar, da BDTD para a coleta de metadados das teses e dissertações diretamente nos RI;

- existência da BDTD como interface separada, tal como existe atualmente, ou ainda como um conjunto de dados pesquisáveis dentro do OASISBr.

\section{REFERÊNCIAS}

ALMEIDA, Maria do Rosário Guimarães. Literatura cinzenta: teoria e prática. São Luís: UFMA, 2000.

DI CESARE, Rosa; SALA, Cesare. The use of grey literature in the agricultural economics field: a quantitative analysis. In:

INTERNATIONAL CONFERENCE ON GREY LITERATURE, 2., 1995.

Proceedings... Washington DC: Transatlantic, 1995. p. 157-168.

OPENDOAR. The Directory of Open Access Repositories. 2015.

Disponível em: <http://www.opendoar.org/>.Acesso em: 3 ago. 2011.

VAN DER GRAAF, Maurits; VAN EIJNDHOVEN, Kwame. Inventory study into the present type and level of OAl compliant digital repository activities in the EU. Amsterdam: Amsterdam University Press, 2008. Disponível em: <http://dare.uva.nl/document/93725>. Acesso em: 3 ago. 2011. 


\section{Title}

Electronic theses and dissertations management in Brazil: about digital libraries of theses and dissertations and institutional repositories

\section{Abstract}

Introduction: This paper presents analysis on the management of electronic theses and dissertations in the Brazilian context. The dilemmas of digital library of theses and dissertations and institutional repositories coexistence and its implications are discussed and possible paths to be considered by actors, in the institutional and national level.

Objective: Present and analyze implications of the digital library of theses and dissertations and institutional repositories coexistence as management systems and, moreover, propose and discuss alternatives faced by actors responsible for its management at the institutional and national levels.

Methodology: Data on the status of theses and dissertations management systems were obtained by IBICT. The analysis allowed to group the Brazilian institutions in three major categories that represent the current state of management of theses and dissertations in Brazil.

Results: The settings were obtained from three major scenarios and their institutional and national implications for the management of theses and dissertations. Of these settings were listed alternative solutions to the problems identified.

Conclusions: Among the findings stand out: the continuity of the IBICT's Brazilian Digital Library of Theses and Dissertations (BDTD) does not necessarily imply the continuity of the digital library of theses and dissertations; Investment in institutional repositories does not mean the end of BDTD; Part of Brazilian universities and research institutions do not enjoy conditions that allow them to effectively maintain the two systems.

Keywords: Theses and dissertations. Electronic theses and dissertations. BDTD. Digital library of theses and dissertations. Institutional repositories. IR. Open access.

\section{Titulo}

Gestión de tesis y disertaciones electrónicas en Brasil: acerca de bibliotecas digitales de tesis y disertaciones y repositorios institucionales

\section{Resumen}

Introducción: Proporciona un análisis de la gestión de las tesis y disertaciones electrónicas en el contexto brasileño. Se discuten los dilemas de la convivência entre las bibliotecas digitales de tesis y disertaciones y repositorios institucionales y sus implicaciones y se propone posibles caminos a ser considerados por los actores responsables de su gestión institucional y nacional. 
Objetivo: El objetivo de este trabajo es presentar y analizar las implicaciones de la coexistencia de las bibliotecas digitales de tesis y disertaciones y repositorios institucionales como sistemas de gestión de tesis y disertaciones y, por otra parte, proponer y discutir alternativas de solución a los problemas que enfrentan los actores responsables de su gestión a nivel institucional y nacional.

Metodología: Se recogieron datos sobre el estado de los sistemas de gestión de tesis y disertaciones en el IBICT. El análisis permitió crear tres categorías principales de grupos de instituciones brasileñas, que representan el estado actual de la gestión de tesis y disertaciones en Brasil.

Resultados: Hay tres grandes escenarios e implicaciones institucionales y nacionales de la gestión de tesis y disertaciones. A partir de las configuraciones de los escenarios se ha propuesto soluciones alternativas a los problemas identificados.

Conclusiones: Entre los resultados se destacan los siguientes: la continuidad de La Biblioteca Digital Brasileira de Teses e Dissertações (BDTD) no implica necesariamente en la continuidad de las bibliotecas digitales de tesis y disertaciones locales; el fortalecimiento de los repositorios institucionales no significa el término de la BDTD; parte de las universidades brasileñas e instituciones de investigación no gozan de condiciones que les permiten mantener con eficacia los dos sistemas.

Palabras clave: Tesis y disertaciones. Tesis y disertaciones electrónicas.

BDTD. Biblioteca digital de tesis y disertaciones. Repositorios institucionales.

RI. Acceso abierto.

Recebido em: 01/03/2015

Aceito em: 21/08/2015 